\title{
Laser for colorectal polyps and cancer
}

\author{
R LAMBERT, MD
}

\begin{abstract}
Laser photodestruction of colorectal polyps and cancer applies almost exclusively to neoplastic lesions. The best results are obtained in exophytic, noninfiltrative, noncircumferential tumours. Neodymium YAG (Nd:YAG) laser is the usual source, and sessions may be repeated at intervals of three to four days during tumour reduction, and at intervals of four to 12 weeks during maintenance therapy. Hemorrhage is a frequent but not severe complication; perforation is the potentially more severe complication. With experience, laser treatment complications decrease to less than $1 \%$. The most common indication of laser photodestruction concerns destruction of colorectal adenomas. Most indications in malignant adenomas are restricted to the rectum. Can J Gastroenterol 1990;4(9):546-548
\end{abstract}

Key Words: Colorectal adenoma, Colorectal cancer, Endoscopy, Laser

\section{Le laser pour les polypes et le cancer recto-coliques}

RESUME: La photodestruction au laser des polypes et cancers recto-coliques est réservée presque exclusivement aux lésions néoplasiques. Les meilleurs résultats sont obtenus dans le cas des tumeurs exophytes, non infiltrantes et non circonférentielles. Le laser NdYAG est la source habituelle; les séances peuvent se répéter à trois ou quatre jours d'intervalle durant la réduction tumorale, et toutes les quatre à 12 semaines au cours du traitement d'entretien. L'hémorragie est une complication fréquente quoique sans gravité, la perforation étant une complication potentiellement plus sévère. Lorsque le traitement est administré par des mains expérimentées, les complications n'atteignent pas 1\%. L'indication la plus commune de la photodestruction au laser est l'adénome colo-rectal. La plupart des indications concernant les adénocarcinomes sont limitées au rectum.

\section{$\mathrm{L}$} ASER PHOTODESTRUCTION OF colorectal polyps and cancer applies almost exclusively to neoplastic lesions, including adenomatous polyps with tubular or villous structure and adenocarcinoma (1). Very few indications concern non-neoplastic polyps (juvenile or hyperplastic) or nonepithelial tumours. Most procedures are carried on during the course of flexible colonoscopy; however, treatments in the low rectum may be performed through the rigid proctoscope or with anal dilation.

The relief of symptoms of colorectal polyps (bleeding, rectal discharge,

\section{OBJECTIVES OF TREATMENT}

Gastroenterology Unit and INSERM U 45, Lyon, France

Correspondence and reprints: Dr R Lambert, Gastroenterology Unit and INSERM U 45 , Hospital E, Herriot 69437, Lyon Cedex 03, France obstruction) is achieved through mini. mal destruction of the tumour mass without interfering with its natural evolution and the lifespan of the patient. This means that persistence of symptoms, or occurrence of any com. plication is a failure.

The objective of subtotal destruc. tion of the tumour is not limited to the relief of symptoms. An extensive destruction is performed in order to ex. tend the length of evolution and patient lifespan. This radical treatment achieves a curative result in some patients.

The objective of total destruction of the tumour is curative. This means that incomplete destruction (or recurrence) is a failure. Complete destruction is pos. sible for an epithelial tumour when there is no argument for an associated malignant focus. The rationale for complete destruction of a malignant lesion is acceptable when there are sound ar. guments for its superficial character (not outpassing the submucosa) and the integrity of lymph nodes. These arguments are difficult to obtain in a preoperative staging.

\section{ENDOSCOPIC PATTERN OF THE TUMOUR}

The best results are obtained in exo. phytic, noninfiltrative, noncircum. ferential tumours. Such characters apply to most but not all polyps. Indeed, some villous adenomas spread like a carpet on a large surface, and extend to the luminal circumference. Any local 
procedure of destruction will generate a more or less severe degree of stenosis.

The majority of adenocarcinomas do not fulfill the favorable criteria. Infiltration and circumferential extension are drastic limiting factors in treatment efficacy. Extension in depth rules out any complete destruction through a conservative procedure if the invasion involves the full thickness of the muscularis propria; furthermore, there is an increased risk of perforation.

\section{ASSESSMENT OF MALIGNANCY}

In confirmed cancer, preoperative staging of the tumour is usually incomplete; both preoperative staging and patient operability should, however, always be attempted when examining the alternatives: surgical resection versus 'conservative' treatment. As yet, endosonography is the most appropriate method of staging the parietal extension of the tumour; it is adapted to the rectum with a rigid probe and better results are obtained with the echoendoscope, allowing exploration in the sigmoid or even proximal colon.

in presence of a polyp nonresectable with a snare, classification is based on repeated forceps biopsies (before, during and after treatment). Classification of the degree of dysplasia of an adenomatous polyp as light, moderate or severe is rather easy. On the other hand, detection of focal cancer is less effective. Furthermore, assessment of the degree of penetration of the malignant cell in the digestive wall is uncertain in the absence of an operative specimen. In the rectum, nonoperative staging is possible, based upon palpation and endosonography. Most difficulties occur with the villous type of adenoma (high malignant potential, inaccurate endosonographic staging).

\section{METHODOLOGY}

Colonoscopy: The preparation is as usual - oral cleansing (salinepolyethylene glycol solution) when the lesion to be treated is proximal to the midsigmoid, and enema when the lesion is located in the rectum or low sigmoid. The efficacy of destruction depends upon good control of colonic peristalsis; therefore, neuroleptanalgesia is required for treatment in the proximal colon and even for long sessions in the rectum.

Laser sessions: The neodymium YAG (Nd:YAG) is the usual laser source (over 90\% of indications). The laser beam (near infrared) is transported through a flexible quartz fibre in the operative channel of the colonoscope. A bare fibre is easier to use (no gas insufflation, no repair of the tip). The photodestruction is powerful ( 30 to 80 W) and penetrates deeply into the digestive wall. Coagulation is excellent, and the procedure does not generate bleeding, even in patients with a deficient hemostasis. The increase in tissue temperature generates pain in the rectum. Laser sessions may be repeated at intervals of three to four days during the acute phase of tumoral reduction, and at intervals of four to 12 weeks during maintenance therapy.

The carbon dioxide laser is adapted to the destruction of small adenomatous areas in the low rectum, near the anal margin. The laser beam, transported by mirrors, requires rigid instrumentation. Destruction is more superficial, more accurate and less painful. Hemostasis is poor. The dye laser produces a red beam $(630 \mathrm{~nm})$ adapted to photodynamic therapy. This is still an experimental procedure in the treatment of large and flat villous adenomas located in the rectum.

\section{COMPLICATIONS}

Hemorrhage is a frequent but not severe complication. It may occur during the laser session or after an interval of 24 to $72 \mathrm{~h}$. Infection, local or systemic, is effectively prevented by prophylactic antibiotic therapy when the irradiation is located in the rectum. Perforation is potentially the more severe complication. Management depends upon the type of colon cleansing (complete or partial), the site of perforation (extra- or intraperitoneal) and size. According to symptoms arising during the first hours after the complication, the treatment is conservative (parenteral nutrition, aspiration, antibiotics) or surgical. Infection is effectively prevented by antibiotic prophylaxis. In laser treatments the number of complications decreases with experience to an acceptable level (severe complications not more than $1 \%$ ) (2).

\section{INDICATIONS}

Adenocarcinoma: Most indications concern partial destruction for palliation of symptoms in patients with a nonoperable cancer. Lesions are located in the rectum or low sigmoid, but not above. Contraindication to surgery (resection) includes advanced age and nonacceptance of colostomy. All possibilities for surgical treatment (including the colo-anal anastomosis) should be explored prior to adoption of 'conservative treatment.' In a patient in good health, the presence of liver metastases is not always a contraindication to surgery. Laser photodestruction is well adapted to the relief of symptoms such as bleeding or rectal discharge but not intestinal obstruction.

Subtotal destruction by laser is seldom considered as a substitute for surgery; indeed, a complete result is uncertain. Therefore, this type of protocol is proposed when destruction is easy (exophytic, noncircumferential tumour) in a nonoperable patient.

Total destruction (curative objective) is restricted to very few cases: when a small adenocarcinoma (less than $3 \mathrm{~cm}$ ) in the low rectum is nonulcerated and well differentiated with limited penetration in the muscular layer; and in a patient accepting a strict surveillance program and eventual reconsideration of the surgical indication.

Polyps: The usual treatment for colorectal polyps is resection with a diathermic snare; this allows histological typing of the resected specimen. In about 5 to $10 \%$ of cases the procedure is either dangerous or impossible. Most of these patients can be treated adequately by laser; however, in some cases (very large or multiple tumours) segmental or total colonic resection may be the appropriate decision. The arguments for endoscopic laser treatment of a polyp are: polyp with a very large stalk or a diameter greater than $3 \mathrm{~cm}$; and sessile adenoma-altered hemostasis. A 
number of parameters are taken into consideration prior to laser treatment. Location of the lesion: Around $60 \%$ of indications are in the rectum (easy endoscopic approach and aiming of laser beam, destruction possible in depth in the extraperitoneal sector). Sessile villous adenomas, 2 to $3 \mathrm{~cm}$ in diameter, are an elective indication, being destroyed in a single session. Large adenomas ( 3 to $6 \mathrm{~cm}$ ) require repeated sessions for their destruction; therefore, an alternative one step surgical transanal tumorectomy is also possible. Flat, circumferential adenomas spreading to most of the rectal surface will be destroyed progressively by laser sessions in a 12 to 18 month program. The treatment could be shortened to an initial step of photodynamic therapy. The surgical alternative in a young patient is complete rectal resection with coloanal anastomosis. The presence of focal cancer in the adenoma is not a contraindication to laser photodestruction, but very careful follow-up is required. In case of failure of destruction, the surgical alternative is reconsidered.

In the sigmoid ( $20 \%$ of indications), sessile polyps are treated by laser, but large or circumferential lesions should always be treated by surgery. Focal cancer in the polyp is an argument for surgery.

In the proximal colon ( $20 \%$ of indications), sessile adenomas under $2 \mathrm{~cm}$ in diameter are adequately treated by laser, as are diminutive polyps (less than $0.5 \mathrm{~cm}$ ). Polyps over $2 \mathrm{~cm}$ are not a good indication and the surgical alternative is preferred.

Number of lesions: Laser treatment is well adapted to treating isolated polyps and some patients with multiple polyposis (more than 10). However, when the number is greater than 30 the surgical alternative (colectomy) may be less aggressive than multiple endoscopic preparations and explorations. In diffuse hereditary adenomatous polyposis, the indications for laser are limited to surveillance after colectomy and colorectal anastomosis, for clearance of all diminutive polyps in the rectal area.
Histology: Most indications concern neoplastic adenomatous polyps. Caution in treatment and surveillance of results is the rule when the architecture is the villous type. Indeed, the malignant potential of such a lesion is higher. Photodestruction of a villous adenoma with demonstrated malignancy at biopsy is possible if tumour depth is superficial as demonstrated in echoendoscopy (T1 stage).

In a few cases, laser photodestruction is prescribed to control the bleeding from juvenile polyps located in the rectum. Treatment of nonepithelial tumours (carcinoids, lymphoma) is not recommended.

\section{RESULTS OF ENDOSCOPIC LASER THERAPY}

From November 1982 to November 1989 , laser photodestruction of colorectal neoplasia was performed in 1349 patients in the author's gastroenterology unit. Indications concerned the rectum in 765 patients, sigmoid in 376 , proximal colon in 152 and diffuse or multiple polyposis before or after surgery in 56. Partial or extensive tumour reduction was proposed with a palliative objective in 426 patients, of which 316 had rectal cancer. Laser photodestruction with a curative objective was proposed in 876 patients with colorectal adenoma; 144 with focal cancer; 163 with moderate dysplasia; 569 with slight dysplasia.

Adenocarcinoma: Palliation of symptoms is obtained after a short delay for bleeding patients in about $90 \%$ of cases; results for rectal discharge are also good. The patient should then be treated on a maintenance program with sessions at intervals of a few weeks. After six months, the rectal function is altered by a reduction in volume of the rectal ampulla and fibrosis; however, most patients ( $90 \%)$ escape to colostomy during their lifespan.

Polyps: Laser photodestruction of isolated polyps is a simple and effective procedure in common practice in endoscopy centres. The rate of successful destruction is greater than $90 \%$. When lesions are multiple, large or located in the proximal colon, the protocol will require repeated endoscopies; the surgical alternative should be considered in due time.

A careful surveillance should be maintained after destruction; indeed, following destruction the cumulative rate of recurrence is high, reaching around $35 \%$ in two years. Such lesions occur at the site of the lesion or in their vicinity. They are easily destroyed during the surveillance program.

When the malignancy is present in a sessile villous adenoma before destruction, the surveillance program should be very strict and prolonged over five years. Recurrence at an early stage is adequately treated by repeated laser sessions if benign. On the other hand, malignant recurrence means failure of conservative treatment; surgery should be considered. Late malignant recurrence may occur in some patients after a few years of negative follow-up. Echoendoscopy should now be included in the surveillance program.

\section{CONCLUSIONS}

Laser photodestruction in colorectal neoplasia is proposed in about one-third of indications for palliation of symptoms from advanced rectal cancer. The most common indication concerns the destruction of colorectal adenomas; most of them being sessile and without demonstrated malignancy. Demonstration of a malignant focus on the forceps or snare biopsies is not a contraindica. tion to laser treatment, if endoscopic access for treatment and surveillance is easy. Therefore, most indications in malignant adenomas are restricted to the rectum.

\section{REFERENCES}

1. Mathus-Vliegen EMH, Tytgat GNJ. Colorectal adenoma. In: Riemann JF, Ell C, eds. Lasers in Gastroenterology. New York: Thieme Publishers, 1989:72-80.

2. Mathus-Vliegen EMH, Tytgat GNJ. Analysis of failures and complications of neodymium; YAG laser photocoagulation in gastrointestinal tract tumors. Endoscopy 1990;22:17-23. 


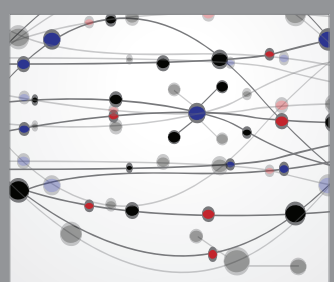

The Scientific World Journal
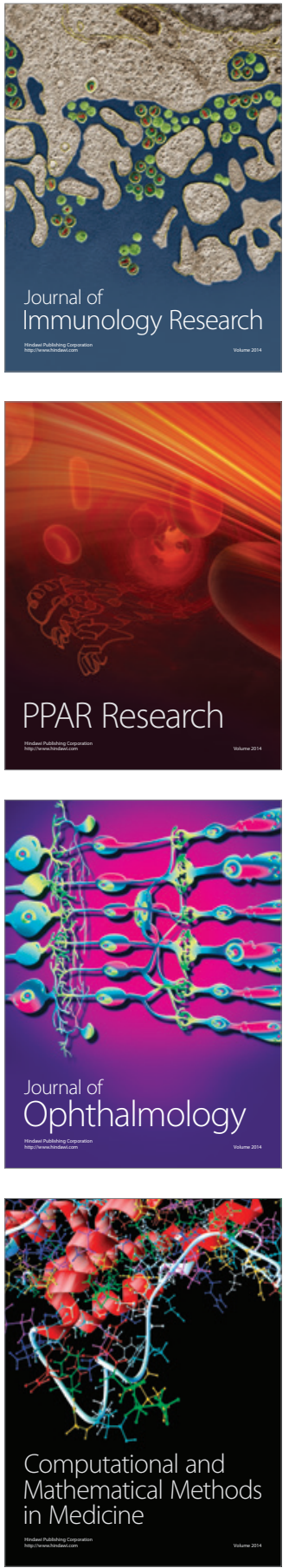

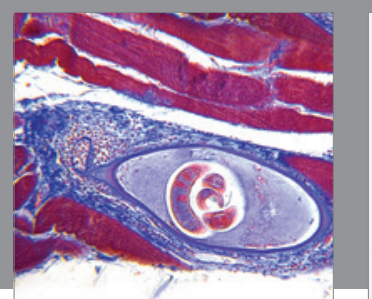

Gastroenterology Research and Practice

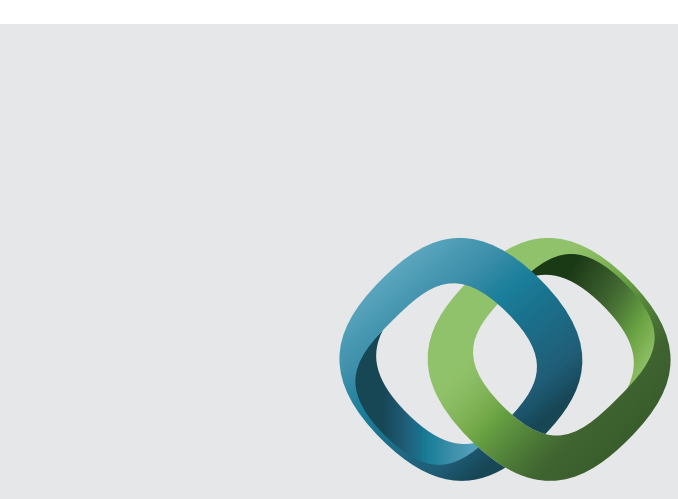

\section{Hindawi}

Submit your manuscripts at

http://www.hindawi.com
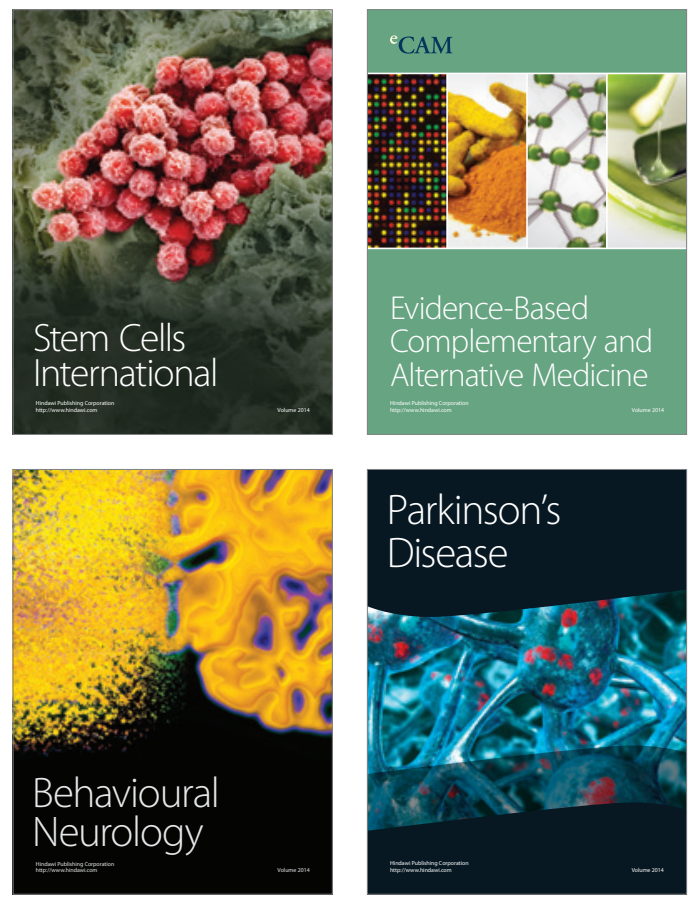
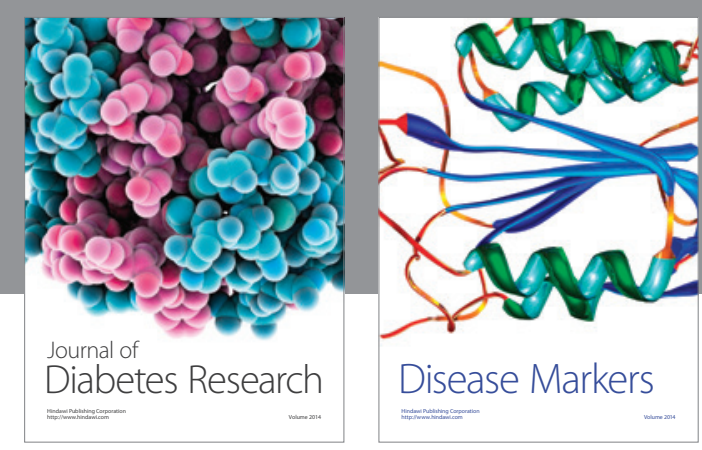

Disease Markers
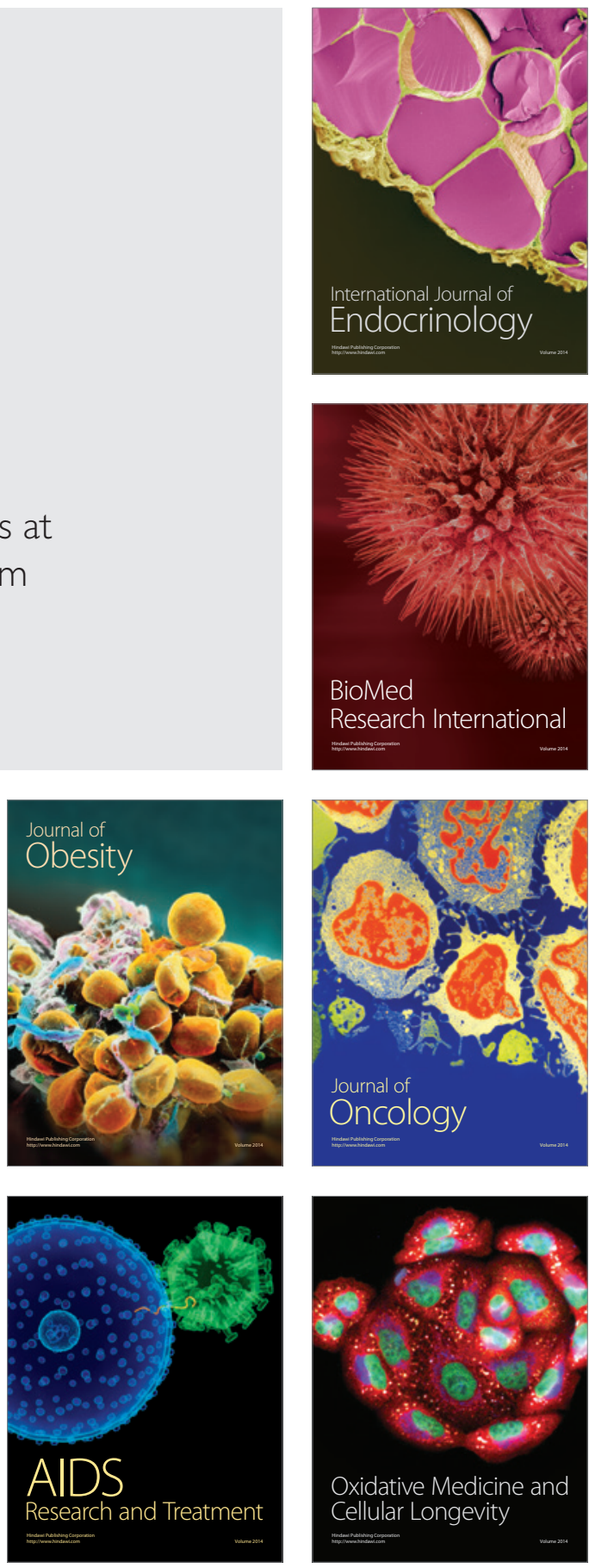